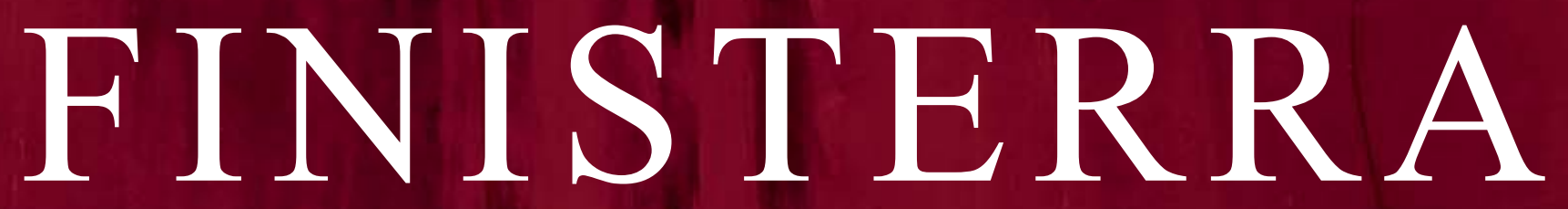

Revista Portuguesa de Geografia

Centro

de Estudos

Geográficos

Volume LII

Número 106

Dezembro 2017 


\title{
TEORIAS NÃO-REPRESENTACIONAIS NA GEOGRAFIA I: CONCEITOS PARA UMA GEOGRAFIA DO QUE ACONTECE
}

Daniel Paiva $^{1}$

\begin{abstract}
RESUMO - As teorias não-representacionais são incontornáveis na geografia cultural contemporânea, pelo que se justifica uma revisão crítica dos principais contributos que tem fornecido à disciplina. Com isso em mente, o objetivo deste artigo é abordar o desenvolvimento das teorias não-representacionais na geografia, e providenciar uma leitura crítica do seu impacto na geografia cultural. As teorias não-representacionais podem ser caracterizadas como uma perspetiva teórica que foca os aspectos processuais e dinâmicos do quotidiano - as práticas, as experiências, as performances - procurando ir para além de leituras segmentadas da realidade e da experiência humana. Para atingir o propósito do artigo, abordarei as genealogias teóricas das teorias não-representacionais e os seus principais fundamentos em três partes: (i) subjetividade, afetos, e sensações; (ii) materialismo relacional; e (iii) movimento, tempo, e eventos.
\end{abstract}

Palavras-chave: Teorias não-representacionais; geografia cultural; afeto; assemblage; evento.

ABSTRACT - NON-REPRESENTATIONAL THEORIES IN GEOGRAPHY I: CONCEPTS FOR A GEOGRAPHY OF WHAT HAPPENS. Non-representational theories are central in contemporary cultural geographies, and for this reason a critical review of its contributions is pertinent. With that in mind, the objective of this article is to approach the expansion of non-representational theories in geography, and to provide a critical reading of its impact in cultural geography. Non-representational theories may be characterized as a theoretical perspective that focuses on the processual and dynamic aspect of everyday life - practices, experiences, and performances - and that seeks to go beyond segmented analysis of reality and human experience. In order to reach this article's goal, I will approach the genealogy of non-representational theories and their main concepts in three segments: (i) subjectivity, affect, and sensation; (ii) relational materialism; and (iii) movement, time, and events.

Keywords: Non-representational theories; cultural geography; affect; assemblage; event.

Recebido: outubro 2016. Aceite: fevereiro 2017.

1 Investigador Associado do Centro de Estudos Geográficos do Instituto de Geografia e Ordenamento do Território da Universidade de Lisboa, R. Branca Edmée Marques, 1600-276, Lisboa, Portugal. E-mail: daniel.paiva@campus.ul.pt 
RÉSUMÉ - LES THÉORIES NON-REPRÉSENTATIONNELLES EN GÉOGRAPHIE I: DES CONCEPTS ADAPTÉS À UNE GÉOGRAPHIE DE «CE QUI SE PASSE». La Géographie culturelle contemporaine ne peut ignorer les théories non-représentationnelles. C'est pourquoi on présente ici l'évolution de leur usage en Géographie ainsi qu'une lecture critique de leur influence sur la Géographie culturelle. Ces théories apportent une vision nouvelle sur le processus et la dynamique du quotidien, sur les usages, les expériences et leurs résultats. On essaie ainsi de dépasser une présentation parcellaire de la réalité et des expériences humaines. Pour cela, on présente les généalogies de ces théories et leurs bases principales : 1. la subjectivité, l'affect et les sensations, 2. le matérialisme relationnel, 3. le mouvement, le temps, les événements.

Mots clés: Théories non-représentationnelles; Géographie culturelle; affect; assemblage; événement.

\section{INTRODUÇÃO}

Desde o início do século, as teorias não-representacionais têm tomado uma posição cada vez mais influente na geografia cultural. A sua expansão está ligada a um interesse crescente entre os geógrafos em perceber e explicar os aspetos processuais e performativos da vida quotidiana, criando uma "geografia do que acontece" (Thrift, 2008, p. 2). Na sequência do interesse da geografia sobre as estruturas sócio-espaciais (Harvey, 1973), a identidade pessoal em relação ao lugar (Tuan, 1977) ou o papel dos símbolos e dos discursos (Soja, 1996), o interesse das teorias não-representacionais passa a ser compreender os processos da vida quotidiana e explicar como os espaços, estruturas, práticas, identidades e relações sociais produzem experiências e afetações no dia a dia que potenciam ou são geradas pelos acontecimentos mundanos. Apesar do desenvolvimento das teorias não-representacionais não estar ligado apenas à Geografia, com contribuições significantes de outros cientistas sociais (Vannini, 2015), é por excelência no seio da Geografia que estas teorias se têm desenvolvido (Lorimer, 2005; Thrift, 2008; Anderson \& Harrison, 2010), muito devido ao facto de que as relações entre o ser humano e todo o ambiente que o rodeia são a preocupação central da própria disciplina.

O objetivo deste artigo é discutir os principais fundamentos das teorias não-representacionais ${ }^{i}$. As teorias não-representacionais têm como foco a experiência e a prática da vida quotidiana, mas pretendem analisar mais do que aquilo que é conscientemente percepcionado pelos sujeitos (Thrift, 2008). Para isso, as teorias não-representacionais trabalham com um entendimento alargado do sujeito e da sua capacidade de ação, considerando-os como relacionais, sendo a sua ação potenciada ou constrangida pelas materialidades com que se relaciona (Anderson \& Harrison, 2010; Simpson, 2015). Nesse âmbito, são os fluxos afetivos entre corpos e materialidades que combinam aspetos de significação representacional e aspetos performativos não-representacionais que são principalmente focados (McCormack, 2013; Anderson, 2014). É justamente este foco nos aspetos que estão para além da representação na performatividade do dia a dia que levam ao desígnio de 'teoria não-representacional'. No entanto, este mesmo desígnio tem sido sujeito a críticas, visto que o foco em aspetos não-representacionais não implica a exclusão da representação, que é fundamental na performatividade do dia a dia, levando alguns autores a propor a designação 'mais-do-que-representacional' (Lorimer, 2005). Este foco tende a esbater alguns dos limites tradicionais nas ciências sociais, como as divisões social/biológico ou mente/ corpo, alargando assim o espetro de análise do que é 'cultural', passando agora a incluir o biológico, o natural, e o físico. 
Este artigo divide-se nas seguintes secções. Primeiro, começarei por discutir as genealogias teóricas das teorias não-representacionais. De seguida, abordarei os seus principais fundamentos em três partes: (i) subjetividade, afetos, e sensações; (ii) materialismo relacional; e (iii) movimento, tempo, e eventos. Por último, farei um breve comentário sobre o impacto desta viragem conceptual.

\section{UMA GENEALOGIA DO NÃO-REPRESENTACIONAL NA GEOGRAFIA}

Anderson e Harrison (2010) argumentam que as teorias não-representacionais emergem na Geografia Humana principalmente na sequência da influência do construtivismo social durante os anos 80 e 90 do século passado. Para estes autores, o construtivismo social foi o modo dominante de análise social e cultural na Geografia Humana, sendo o principal fundamento teórico do que ficou conhecido como a Nova Geografia Cultural. Neste campo, o interesse de investigação incidia principalmente numa questão de representação, e em especial no que respeita às estruturas de significado simbólico de uma sociedade ou cultura. São seminais neste aspeto os trabalhos sobre paisagens de Cosgrove e Daniels (1988), Duncan (1990) e Zukin (1991). Os trabalhos influenciados pelo construtivismo social, para Anderson e Harrison (2010), deixaram na Geografia Cultural duas ideias-chave. Primeiro, que as ordens simbólicas societárias são socialmente construídas e arbitrárias. Em segundo lugar, que a interpretação destas mesmas ordens simbólicas é plural e contestada, e difere de lugar para lugar. Poder-se-ia acrescentar uma terceira ideia-chave, que já está na ponte entre o interesse da Nova Geografia Cultural nas ordens simbólicas e o interesse das teorias não-representacionais na performance política, que é a importância das dinâmicas de poder e influência das estruturas simbólicas na prática quotidiana (Agnew, 1987; Massey, 1994).

Voltando à lógica de Anderson e Harrison (2010), os mesmos afirmam que as teorias não-representacionais rompem com o construtivismo social ao criticar a separação que este faz entre o mundo e os significados que lhe são atribuídos. As teorias não-representacionais vêm contestar essa divisão, afirmando que o conhecimento não é divisível da realidade no qual emerge, contrariando a clássica divisão cartesiana entre representação e realidade, entre mente e corpo. Neste sentido, a produção de conhecimento passa a preocupar-se não apenas com as representações mentais da realidade (e.g. significados, sentimentos, discursos, estruturas), mas também com o modo como essas representações são produzidas e praticadas corporeamente no decorrer das acções e interacções de que o mundo é constituído (incluíndo práticas, hábitos, afetos). A performance torna-se assim numa palavra-chave.

Um momento-chave nesta transição é a crítica de Thrift e Dewsbury (2000) às 'geografias mortas'. Num seminal editorial na revista Environment and Planning $D$, os autores argumentaram que as análises geográficas dominantes nesse momento, assentes na representação, apresentavam a realidade geográfica como uma realidade estática e imutável, negligenciando a natureza contínua e processual dos fenómenos geográficos, e traçaram as principais linhas do que deveria ser uma geografia performativa, com grande ênfase no não-representacional.

Mas a evolução das teorias não-representacionais na Geografia Humana não pode ser apenas atribuída ao construtivismo socialii. O impacto da filosofia pós-estruturalista (Doel, 2010) e da pós-fenomenologia (Ash \& Simpson, 2014) são inescapáveis quando se fala de teorias não-representacionais. Neste sentido, as teorias não-representacionais podem também ser vistas como descendentes da linhagem crítica das geografias estruturalistas e da linhagem hermenêutica das geografias humanistas que se desenvolveram na década de 1970, embora configurem os temas de pesquisa de um modo diferente. Por um lado, se o interesse nas estruturas sociais permanece, o foco passa agora por perceber como é que essas estruturas sociais afetam a ação social (Anderson, 2014). Por outro lado, o interesse na subjetividadee na percepção dosfenómenos pelos indivíduos permanece central nas teorias não-representacionais, 
mas trabalhos recentes no âmbito da pós-fenomenologia têm alterado a maneira como se aborda esses aspetos. Enquanto a geografia humanista de base fenomenológica se focava na representação mental do mundo pelo sujeito, as teorias não-representacionais de base pós-fenomenológica abordam o modo como o sujeito em si emerge relacionalmente com o mundo (Ash \& Simpson, 2014).

Importa também salientar a importância da teoria ator-rede, desenvolvida por sociólogos como Law (2004) e Latour (2005), e cujos preceitos significantes para a geografia foram essencialmente absorvidos pelas teorias não-representacionais. A noção alargada de agênciaiii que Latour (2005) defende é talvez a peça central desta absorção. As reflexões de Latour (2005) foram particularmente felizes em descentralizar a noção de agência do ser humano, passando-se a considerar a agência como um fluxo no qual participam não só humanos (e seres vivos não-humanos), mas também diversos actantes, nomeadamente objetos, tecnologias ou a própria morfologia do espaço. Esta perspetiva tem sido particularmente útil em estudos urbanos para analisar a ação social em relação com o espaço e os seus objetos (Bender \& Farías, 2010; McFarlane, 2011).

Estas três correntes (filosofia pós-estruturalista, filosofia pós-fenomenológica, e teoria ator-rede) têm em comum a destabilização de noções fundamentais como a de estrutura, sujeito, corpo, ou agência, passando a entendê-los de uma maneira fluída e processual, e é por esse motivo que se tornaram instrumentais para o objetivo de focar os aspetos performativos da experiência do mundo. Na próxima secção, discutir-se-ão os principais fundamentais das teorias não-representacionais na Geografia, abordando-se alguns fundamentos teóricos, nomeadamente: (i) subjectividade, afetos e sensações; (ii) materialismo relacional; e (iii) movimento, tempo e eventos.

\section{SUBJETIVIDADE, AFETOS E SENSAÇÕES}

A noção de sujeito e subjetividade é central para um programa de investigação focado na experiência performativa do mundo. No entanto, a relevância dos processos não-representacionais nessa experiência performativa obrigaram a uma reformulação da noção de sujeito e subjetividade (Wylie, 2010; Simpson, 2015).

Numa primeira instância, as teorias não-representacionais vêm destabilizar e descentralizar o sujeito. Esta descentralização tem várias origens, desde as reflexões no campo da filosofia pós-estruturalista (Deleuze, 1988) e da pós-fenomenologia (Böhme, 1995; Stiegler, 1998; Nancy, 2007) a novas teorias da psicologia social (Brennan, 2004). Têm estas em comum a noção que não é possível, ou pelo menos não é producente, separar em absoluto o sujeito e o seu exterior, bem como não o é separar a mente (ou psicologia) e o corpo (ou fisiologia) do sujeito. O conceito comum que articula estas noções tem sido o conceito de afeto ${ }^{\text {iv }}$. A leitura mais tradicional de afeto será a de Espinosa, que é recuperada e repensada por Deleuze (1988), sendo afeto definido como o conjunto de capacidades de um organismo para afetar outros organismos e ser ele próprio afetado. Pensar a partir da noção de afeto implica perceber que a ação humana não é apenas determinada pelo pensamento consciente. Ela é também determinada por vários processos não-representacionais, como sensações ou sentidos, sentimentos, pulsões, hábitos, reflexos fisiológicos automáticos, entre outros (Massumi, 2002; Thrift, 2008). O sujeito é constantemente afetado por diversos estímulos ambientais percebidos pelo sistema neurológico, e as teorias não-representacionais na Geografia têm focado esta interacção constante que coloca em causa a ideia de um sujeito contido, isto é, que o sujeito se resume ao seu corpo e que este pode ser entendido sem se entender o ambiente em que vive (Brennan, 2004; Thrift, 2008). A partir da leitura do afeto, esbatem-se as distinções entre o social e o biológico, entre cultura e natureza, e entre mente e corpo (Brennan, 2004).

Neste âmbito, tem tido particular importância para a geografia o conceito de atmosferas afetivas, o qual tem potenciado vários estudos empíricos (Bissel, 2010; Edensor, 2012; Ash, 2013b; Bille, 2015). 
Anderson (2014) define atmosferas afetivas como o que está "entre" no espaço urbano, o terreno partilhado onde os afetos se transmitem. No entanto, estes não se transmitem apenas entre corpos. Como Thrift afirma, a transmissão afetiva é também "uma propriedade de espaços particulares embebidos num afeto ou numa combinação de afetos ao ponto de espaço e afeto serem regularmente coincidentes" (2008, p. 222), isto é, as materialidades têm um papel relevante. Para autores como Stewart (2011), os indivíduos são invariavelmente forçados a sintonizarem-se com as atmosferas dos lugares onde se encontram, pelo impacto no seu corpo.

Apesar disto, tem sido argumentado que descentralizar completamente a agência do sujeito será ir longe demais. Tendo em conta que os processos não-representacionais funcionam em diálogo com o pensamento reflexivo (Long \& Moore, 2012; Blackman, 2012), tem-se estabelecido um entendimento do sujeito enquanto sujeito relacional mas reflexivo, enquanto presença autociente no mundo (Wylie, 2010; Simpson, 2015).

Na sequência da importância dada aos processos que estão para além da representação, têm surgido vários estudos que se debruçam sobre sentidos menos explorados, como a audição (Duffy \& Waitt, 2011; Gallagher \& Prior, 2014), o tacto (Paterson, 2009) ou o olfacto (Henshaw, 2014), emergindo assim uma linha de investigação geográfica sobre paisagens sensoriais, ou sensescapes (Degen, 2008; Middleton, 2010), que dá seguimento ao interesse da geografia humanista sobre sentidos (Tuan, 1977), e que se interliga com uma sensorial turn ao nível das ciências sociais que reformula métodos etnográficos para o estudo social, antropológico e geográfico dos sentidos (Howes, 2005; Pink, 2009; Pink, 2013).

\section{MATERIALISMO RELACIONAL}

Relacionado com a descentralização do sujeito e da agência, e com a perspetiva afetiva sobre o corpo, está uma maior importância dada ao papel das materialidades na ação social. O conceito mais central deste materialismo relacional será o de assemblage. Esta importância é herdada da teoria ator-rede e da sua consideração do 'social' não como uma entidade pré-dada, mas como formações ou associações na qual participam não só humanos (denominados atores), mas toda uma série de entidade não-humanas (denominadas actantes), como textos, objectos, tecnologias, entre outros (Latour, 2005). Assemblage, portanto, refere-se à mobilização, ou encontro, de vários atores e actantes na ação social. Todas as formações sociais são vistas como uma montagem de elementos específicos que determinam a ação social através do seu relacionamento. Para além do pensamento de Latour (2005), é também a definição de Deleuze e Guattari (1987) de assemblage que tem sido mobilizadav . Esta tem a vantagem de os filósofos considerarem dois níveis em cada assemblage: um nível de atividade, de formação física, e um nível de expressão, associado aos fenómenos afetivos.

A participação de diferentes materialidades na ação social tem sido considerada de diferentes modos na Geografia (Anderson \& Wylie, 2009), não sendo possível classificar de forma simplista esses modos devido à própria multiplicidade de formas que as materialidades assumem e os modos como elas se diluem na ação social (Anderson \& Harrison, 2010). Mas é possível distinguir duas vertentes nos estudos sobre os modos de relacionamento que estas materialidades provocam.

Uma linha significante de trabalhos que emerge neste contexto é a exploração da agência da morfologia espacial e da arquitectura nas práticas, nas sensações, nos ritmos e na apropriação do espaço. Por exemplo, Kärrholm (2007; 2008; 2009), Amin (2008) e Dewsbury (2015) têm argumentado que a morfologia espacial do espaço público é determinante para a apropriação do espaço na medida em que determinadas materialidades como o mobiliário urbano influenciam a localização e os ritmos dos indivíduos. Por outro lado, outros estudos têm apontado para a influência afetiva da morfologia espacial e as suas variações, como o de Adey (2008) que mostra como espaços como os aeroportos são desenhados 
para induzir uma determinada mobilidade no seu interior, sendo a experiência espacial planeada ao detalhe dos afetos, para assegurar controlo sobre os indivíduos nesses espaços, ou o estudo de Edensor (2012) sobre iluminação no festival Blackpool Illuminations que mostra a participação da luz nas atmosferas afetivas de Blackpool. Outros estudos semelhantes têm analisado centros comerciais (Miller, 2014), edifícios (Kraftl \& Adey, 2008), praias (Lobo, 2014), ou transportes (Bissell, 2010).

Outra linha significante sobre materialidades incide sobre objetos, e em particular novas tecnologias digitais. Destes, destacam-se os estudos de Ash (2012a; 2012b; 2013a) sobre videojogos que mostram como o corpo se adapta e sintoniza com as materialidades das novas tecnologias à medida que as utiliza. Um conceito essencial neste âmbito é o conceito de affordance, apresentado por Gibson (1979) para se referir às possibilidades de relação entre organismos e objetos que são apercebidas através de estímulos que os objetos provocam nos organismos (Thrift, 2008). Mais concretamente, affordances são "convites ou significados ecológicos que estão ontologicamente enraizados em coisas ou quasi-coisas" (Griffero, 2014, p. 51). Simplificando, raramente um objeto é inexpressivo, e as suas qualidades são - nem sempre conscientemente - percebidas pelos sujeitos que daí retiram um possível uso para o objeto. Para Griffero (2014), existem vários tipos de affordances, que vão desde indicações práticas que aferimos dos objetos a estímulos emocionais que deles emanam. Este é um nível que as teorias não-representacionais vêm acrescentar às relações de significação representacional (Thrift, 2008). No entanto, affordances apenas explicam parte da relação performativa não-representacional com o mundo. Como Dewsbury (2015) e Bissel (2015) argumentam, os hábitos corporais têm um papel central na performance do dia a dia, e os vários espaços em que estamos e os objetos que usamos são apropriados e manuseados através de gestos que fazemos automaticamente através de hábitos estabelecidos.

\section{MOVIMENTO, TEMPO, E EVENTOS}

No cerne das teorias não-representacionais está o intuito de perceber os aspetos processuais dos fenómenos, de descrever e explicar o que acontece. Para tal, importa especialmente perceber como é que as relações se desenvolvem na prática, os fluxos e movimentos das coisas e os seus resultados. Esta intenção advém de duas percepções. Primeiro, a ideia de que a própria vida é constituída por movimento, que se tem estabelecido tanto por via da affective turn, cujos principais autores apresentam os fluxos afetivos como movimentos espaço-tempo que alteram constantemente o estado dos corpos envolvidos (Massumi, 2002; Clough, 2007), como por via da ecologia política, que tem apresentado a vida social e política como assemblages vibrantes compostos por movimentos que alteram o estado de coisas constantemente (Bennett, 2010; Goodman, 2010). Estas perspetivas têm animado abordagens vitalistas, as quais se focam na sobreposição de aspetos geoculturais e físico-biológicos na ação social (Pløger 2006; Greenhough, 2010; Pløger, 2015). Por outro lado, a percepção de que no mundo atual os movimentos de pessoas, bens e informação aumentam cada vez mais, e que muitos desses movimentos, especialmente os de informação, passam despercebidos, leva a um maior interesse nos espaços de movimento (Thrift, 2008).

Este interesse espelha também uma transição mais vasta no campo da Geografia no sentido da introdução da variável tempo em vários dos conceitos fundamentais da disciplina. Desde o fim do século XX, a noção de espaço passou regularmente para uma noção de espaço-tempo (Crang, 2012), o lugar passou a ser visto como um evento e não como uma entidade estável (Massey, 2005), e a noção de territorialização tem destabilizado a noção de território enquanto entidade perene (Brighenti, 2010), de modo semelhante a como o conceito de regionalidade se debruça sobre a produção da ideia de região e os processos subjacentes (Campbell, 2016). 
Na sequência deste interesse, as teorias não-representacionais têm explorado e desenvolvidos os conceitos de ritmo. O ponto de partida comum destes estudos tem sido as reflexões de Lefebvre (2004) em torno da ritmanálise. Lefebvre (2004) propôs o estudo da espacialidade a partir dos ritmos, argumentando que a ritmicidade é algo que é comum a tudo: ao humano, ao social, ao biológico, ao espacial. Para o autor, o estudo dos ritmos, começa no corpo, porque é este que os capta, mas o objetivo deve ser perceber como os diferentes ritmos dos elementos espaciais se conjugam para formar conjuntos polirítmicos, sendo necessário compreender as interacções espácio-temporais dentro destes conjuntos. Esta perspetiva tem sido aplicada com especial interesse na área dos estudos urbanos, em particular no estudo da apropriação do espaço público (Simpson, 2008; Lehtuvuori \& Koskela, 2013; Wunderlich, 2013; Paiva, 2016) e na mobilidade urbana (Vannini, 2012), mas também na apropriação de outros espaços como espaços digitais (Ash, 2015) ou lúdicos (Henriques, 2010).

Outro conceito fundamental que tem estruturado estudos não-representacionais é o conceito de evento. Este conceito aponta para o estudo de acontecimentos, focando o modo como a transformação do mundo é feita através de instâncias de agência relacional que incluem elementos humanos e não-humanos, e que produzem efeitos e significados acerca desses efeitos (Shaw, 2012). É particularmente importante como alguns dos elementos dos eventos são capazes de 'forçar' outros elementos, seja através da criação ou destruição de elementos, da afetação ou do controlo (Shaw, 2012). Eventos destacam-se por produzirem invariavelmente significados acerca do que acontece. Neste âmbito, eventos são uma parte importante da pertença dos sujeitos ao mundo e da presença do mundo perante o sujeito, visto que o corpo humano está sempre envolvido num evento e tende a sintonizar-se com ele (Stewart, 2011; Pløger, 2015). Pelo seu foco na transformação social, o conceito de evento aponta também para um modo de compreender como aspetos representacionais ou estruturais como significados sociais ou instituições nascem a partir da ação social e a moldam (Shaw, 2012).

\section{CONCLUSÃO}

As teorias não-representacionais emergiram neste século como uma das principais perspetivas na geografia cultural, assumindo-se como um romper com o momento pós-moderno na geografia (para uma crítica do pós-modernismo a partir das teorias não-representacionais, ver Anderson, 2014). A sua emergência não implica a desconsideração de elementos fundamentais para outras perspetivas no âmbito da disciplina, como estruturas sociais, sentido de identidade ou significados coletivos, mas a leitura destes elementos passa a fazer-se a partir da lente do afeto, o que possibilita um entendimento performativo, relacional e processual destes elementos, sendo o foco alargado para a sua produção, conteúdo e influência na ação social. A geografia a partir das teorias não-representacionais procura observar principalmente o que se move, o que acontece, ou o que muda, afastando-se de leituras que abordam a distribuição de elementos humanos e não-humanos no espaço e o território como estruturas estáveis.

\section{AGRADECIMENTOS}

A investigação conducente a este artigo foi financiada pela Fundação para a Ciência e a Tecnologia através da bolsa de doutoramento para o projeto "Som Urbano: Territórios, Atmosferas Afetivas e Políticas Públicas” (SFRH/BD/108907/2015). O autor agradece ao Professor Herculano Cachinho pela orientação científica e a dois revisores anónimos pelos comentários e sugestões de melhoria. 


\section{REFERÊNCIAS BIBLIOGRÁFICAS}

Adey, P. (2008). Airports, mobility and the calculative architecture of affective control. Geoforum, 39, 438-451.

Agnew, J. (1987). Place and Politics: The Geographical Mediation of State and Society. Nova Iorque: Allen \& Unwin.

Amin, A. (2008). Collective culture and urban public space. City: analysis of urban trends, culture, theory, policy, action, 12(1), 5-24.

Anderson, B. (2014). Encountering Affect. Capacities, Apparatuses, Conditions. Surrey: Ashgate.

Anderson, B., \& Harrison, P. (Ed.) (2010). Taking-place: non-representational theories and geography. Surrey: Ashgate.

Anderson, B., Kearnes, M., McFarlane, C., \& Swanton, D. (2012). On assemblages and geography. Dialogues in Human Geography, 2(2), 171-189.

Anderson, B., \& Wylie, J. (2009). On geography and materiality. Environment and Planning A, 41(2), 318-335.

Ash, J. (2015). Interview with Pasi Väliaho on Video Games and Rhythm. Theory, Culture \& Society, 32(7-8), 291-300.

Ash, J. (2013a). Technologies of captivation: videogames and the attunement of affect. Body \& Society, 19(1), 27-51.

Ash, J. (2013b). Rethinking affective atmospheres: Technology, perturbation and space times of the non-human. Geoforum, 49, 20-28.

Ash, J. (2012a). Technology, technicity, and emerging practices of temporal sensitivity in videogames. Environment and Planning A, 44, 187-203.

Ash, J. (2012b). Attention, videogames and the retentional economies of affective amplification. Theory, Culture \& Society, 29(6), 3-26.

Ash, J., \& Simpson, P. (2014). Geography and post-phenomenology. Progress in Human Geography. Retrieved from: http://phg.sagepub.com/content/ early/2014/08/07/0309132514544806.abstract

Bennett, J. (2010). Vibrant Matter: A Political Ecology of Things. Durham: Duke University Press.

Bille, M. (2015). Lighting up cosy atmospheres in Denmark. Emotion, Space and Society, 15, 56-63.

Bissell, D. (2015). Virtual infrastructure of habit: the changing intensities of habit through gracefulness, restlessness and clumsiness. Cultural Geographies, 22(1), 127-146.
Bissell, D. (2010). Passenger mobilities: affective atmospheres and the sociality of public transport. Environment and Planning D: Society and Space, $28,270-289$.

Blackman, L. (2012). Immaterial Bodies: Affect, Embodiment, Mediation. Londres: Sage.

Böhme, G. (1995). Atmosphäre: Essays zur neuen Ästhetik [Atmosphere: essays on the new aesthetics]. Frankfurt am Main: Suhrkamp.

Brennan, T. (2004). The Transmission of Affect. New York: Cornell University.

Brighenti, A. (2010). On Territorology. Towards a General Science of Territory. Theory, Culture \& Society, 27(1), 52-72.

Campbell, N. (2016). Affective Critical Regionality. Londres: Rowman \& Littlefield.

Cosgrove, D., \& Daniels, S. (1988). The Iconography of Landscape: Essays on the Symbolic Representation, Design and Use of Past Environments. Cambridge: Cambridge University Press.

Clough, P. (2007). The Affective Turn: Theorizing the Social. Durham: Duke University Press.

Crang, M. (2012). Commentary. Temporal ecologies: multiple times, multiple spaces, and complicating space times. Environment and Planning A, 44(9), 2119-2123.

Cresswell, T. (2012). Geographical Thought: A Critical Introduction. Londres: Wiley.

Degen, M. (2008). Sensing Cities Regenerating public life in Barcelona and Manchester. Londres: Routledge.

Deleuze, G. (1988). Spinoza: Practical Philosophy. São Francisco: City Lights Books.

Deleuze, G., \& Guattari, F. (1987). A Thousand Plateaus. Capitalism and Schizophrenia. Londres: University of Minnesota Press.

Dewsbury, J. D. (2015). Non-representational landscapes and the performative affective forces of habit: from 'Live' to 'Blank'. Cultural Geographies, 22(1), 29-47.

Doel, M. (2010). Representation and difference. In B. Anderson, \& P. Harrison (Eds.), Taking-place: non-representational theories and geography (pp. 117-130). Surrey: Ashgate.

Duffy, M., \& Waitt, G. (2011). Sound diaries: a method for listening to place. The Journal of Media Geography, VII, 119-136. 
Duncan, J. S. (1990). The city as text. The politics of landscape interpretation in the Kandyan Kingdom. Cambridge: Cambridge University Press.

Edensor, T. (2012). Illuminated Atmospheres: Anticipating and Reproducing the Flow of Affective Experience in Blackpool. Environment and Planning D, 30(6), 1103-1122.

Farías, I., \& Bender, T. (2010). Urban Assemblages. How Actor-Network Theory Changes Urban Studies. Oxon: Routledge.

Gallagher, M., \& Prior, J. (2014). Sonic geographies: Exploring phonographic methods. Progress in Human Geography, 38(2), 267-284.

Gibson, J. (1979). The Ecological Approach to Visual Perception. Boston: Houghton Mifflin.

Goodman, S. (2010). Sonic warfare: Sound, affect, and the ecology of fear. Cambridge, MA: MIT Press.

Greenhough, B. (2010). Vitalist Geographies: Life and the More-Than-Human. In B. Anderson, \& P. Harrison (Eds.), Taking-place: non-representational theories and geography (pp. 37-54). Surrey: Ashgate.

Gregg, M., \& Seigworth, G. (2010). The Affect Theory Reader. Durham: Duke University Press.

Griffero, T. (2014). Atmospheres: aesthetics of emotional spaces. Londres: Ashgate.

Harvey, D. (1973). Social Justice and the City. London: Edward Arnold.

Henriques, J. (2010). The vibrations of affect and their propagation on a night out on Kingston's dancehall scene. Body \& Society, 16(1), 57-89.

Henshaw, V. (2014). Urban Smellscapes. Understanding and Designing City Smell Environments. London: Routledge.

Howes, D. (2005). Empire of the Senses: The Sensual Culture Reader. Oxford: Berg.

Miller, J. C. (2014). Approximating new spaces of consumption at the Abasto Shopping Mall, Buenos Aires, Argentina. Journal of Cultural Geography, 31(2), 206-217.

Middleton, J. (2010). Sense and the city: exploring the embodied geographies of urban walking, Social \& Cultural Geography, 11(6), 575-596.

Kärrholm, M. (2009). To The Rhythm of Shopping - On Synchronisation in Urban Landscapes of Consumption. Social \& Cultural Geography, 10(4), 421-440.

Kärrholm, M. (2008). The Territorialisation of a Pedestrian Precinct in Malmö: Materialities in the
Commercialisation of Public Space. Urban Studies, 45(9), 1902-1924.

Kärrholm, M. (2007). The Materiality of Territorial Production: A Conceptual Discussion of Territoriality, Materiality, and the Everyday Life of Public Space. Space and Culture, 10(4), 437-453.

Kraftl, P., \& Adey, P. (2008). Architecture | Affect | Inhabitation: Geographies of Being-In Buildings. Annals of the Association of American Geographers, 98(1), 213-231.

Latour, B. (2005). Reassembling the Social. An Introduction to Actor-Network-Theory. Oxford: Oxford University Press.

Law, J. (2004). After Method. Mess in social science research. Oxon: Routledge.

Lehtovuori, P., \& Koskela, H. (2013). From Momentary to Historic: Rhythms in the Social Production of Urban Space, the Case of Calçada de Sant'Ana, Lisbon. The Sociological Review, 61(S1), 124-143.

Lobo, M. (2014). Affective energies: Sensory bodies on the beach in Darwin, Australia. Emotion, Space and Society, 12, 101-109.

Long, N., \& Moore, H. (2012). Sociality Revisited: Setting a New Agenda. Cambridge Anthropology, 30(1), 40-47.

Lorimer, H. (2005). Cultural geography: the busyness of being 'more-than-representational'. Progress in Human Geography, 29(1), 83-94.

Massey, D. (2005). For Space. London: Sage.

Massey, D. (1994). Space, place, and gender. Minneapolis: University of Minnesota Press.

McCormack, D. (2013). Refrains for Moving Bodies: Experience and Experiment in Affective Spaces. Durham: Duke University Press.

McFarlane, C. (2011). Assemblage and Critical Urbanism. City: analysis of urban trends, culture, theory, policy, action, 15(2), 204-224.

McFarlane, C. (2009). Translocal assemblages: space, power, and social movements. Geoforum, 40(4), 561-567.

Nancy, J. L. (2000). Being Singular Plural. Stanford, CA: Stanford University Press.

Paiva, D. (2016). Collapsed rhythms: the impact of urban change in the everyday life of elders. Space and Culture, 19(4), 345-360.

Paterson, M. (2009). Haptic geographies: ethnography, haptic knowledges and sensuous dispositions. Progress in Human Geography, 33(6), 766-788. 
Pink, S. (2013). Engaging the Senses in Ethnographic Practice: Implications and Advances. The Senses and Society, 8(3), 261-267.

Pink, S. (2009). Doing Sensory Ethnography. London: Sage.

Pløger, J. (2015). The Evental City: Moment, Situation, Presence. Space and Culture. Retrieved from: http://sac.sagepub.com/content/early/2015/09/0 8/1206331215595729.full.pdf+html

Pløger, J. (2006). In Search of Urban Vitalis. Space and Culture, 9(4), 382-399.

Shaw, I. (2012). Towards an evental geography. Progress in Human Geography, 36(5), 613-627.

Simpson, P. (2015). What remains of the intersubjective?: On the presencing of self and other. Emotion, Space and Society, 14, 65-73.

Simpson, P. (2008). Chronic Everyday Life: Rhythmanalysing Street Performance. Social \& Cultural Geography, 9(7), 807-829.

Soja, E. (1996). Thirdspace: Journeys to Los Angeles and Other Real-and-Imagined Places. Oxford: Basil Blackwell.

Stewart, K. (2011). Atmospheric Attunements. Environment and Planning D: Society and Space, 29(3), 445-453.
Stiegler, B. (1998). Technics and Time 1: The Fault of Epimetheus. Stanford. CA: Stanford University Press.

Thrift, N., \& Dewsbury, J. (2000). Dead Geographies And How to Make Them Live. Environment and Planning D: Society and Space, 18(4), 411-432.

Thrift, N. (2008). Non-representational Theory. Space, Politics, Affect. Oxon: Routledge.

Tuan, Y. F. (1977). Space and Place: The Perspective of Experience. Minneapolis: University of Minnesota Press.

Vannini, P. (2015). Non-Representational Methodologies: Re-Envisioning Research. London: Routledge.

Vannini, P. (2012). In Time, Out of Time: Rhythmanalyzing Ferry Mobilities. Time \& Society, 21(2), 241-269.

Wunderlich, F. M. (2013). Place-Temporality and Urban Place-Rhythms in Urban Analysis and Design: An Aesthetic Akin to Music. Journal of Urban Design, 18(3), 383-408.

Wylie, J. (2010). Non-representational Subjects? In B. Anderson, \& P. Harrison (Eds.), Taking-place: non-representational theories and geography (pp. 99-114). Surrey: Ashgate.

Zukin, S. (1991). Landscapes of Power: From Detroit to Disney World. California: University of California Press.

i Uso aqui o termo 'teorias não-representacionais' no plural (como Anderson \& Harrison, 2010) e não no singular (como Thrift, 2008) porque aquilo que se tem denominado 'Teoria Não-representacional' não é de facto uma teoria, mas uma perspetiva de investigação que mobiliza e produz várias teorias, geralmente relacionadas com aspectos não-representacionais da experiência e prática geográfica (Cresswell, 2012).

ii Anderson e Harrison (2010) admitem e apontam outras possíveis genealogias para as teorias não-representacionais na Geografia, mas não as desenvolvem.

iii Em inglês, agency. Poderá ser traduzido como agência, ou capacidade de ação.

iv Em inglês affect. Em português, afeto está associado a emoções, mas affect é um conceito mais vasto, relacionado com todas as capacidades de um organismo afetar e ser afetado. Para uma genealogia mais vasta do conceito, ver Clough (2007), Thrift (2008), ou Gregg e Seigworth (2010).

v Para outros usos do termo assemblage em Geografia, ver McFarlane (2009) e Anderson, Kearnes, McFarlane e Swanton (2012). 